\title{
Variational Models for Fusion and Denoising of Multifocus Images
}

\author{
Wei-Wei Wang, Peng-Lang Shui, and Xiang-Chu Feng
}

\begin{abstract}
In this letter, variational models in pixel domain and wavelet domain are presented for fusion and denoising of noisy multifocus images. In pixel domain, the problem is formulized as minimizing a weighted energy functional, where the total variation (TV) is used as regularity constraint for noise reduction. A new family of weight functions for fusion is proposed that are based on the local average modulus of gradients and the power transform. In wavelet domain, the problem is formulized as shrinkage of the weighted wavelet coefficients of source images, where weight functions are based on the local average modulus of intra- and interscale wavelet coefficients and the power transform. The experiments are made to verify the effectiveness of the proposed methods.
\end{abstract}

Index Terms-Energy functional, multifocus image fusion and denoising, variational model.

\section{INTRODUCTION}

I MAGE fusion and denoising has become an active issue in image processing owing to the availability of multisensor data in many fields. Due to the limited depth-of-focus of optical lenses in charge coupled devices (CCDs), it is often not possible to get an image that contains all relevant objects "in focus." Several images of the same scene at different focuses are easy to be obtained, and in each image, only the objects "in focus" are clear. Combining several images with different focuses into one uniformly focused image is referred to as the multifocus image fusion. There are a number of methods for multifocus image fusion, and a brief overview is given in [1]. These methods are based on the assumption that images are noise-free. Practically, images are often corrupted by noise in acquisition or transmission. Thus, it is necessary to investigate joint fusion and denoising of noisy multifocus images. Variational methods [2], [3], wavelet methods [4], [8], and their hybrid methods [7] are popular in image restoration. Combining the variational methods [2] and the fusion methods, we present the variational models for joint fusion and denoising of noisy multifocus images in pixel domain and in wavelet domain.

In pixel domain, images are regarded as functions in the bounded variation (BV) space [2], [7]. The proposed variational model can realize both fusion and denoising of noisy multifocus images. Considering existence of noise, we extend the competition fusion rule based on the local average modulus of gradients

Manuscript received May 15, 2007; revised September 11, 2007. The associate editor coordinating the review of this manuscript and approving it for publication was Dr. Zhou Wang.

W.-W. Wang and X.-C. Feng are with the Department of Mathematics, Xidian University, Xi'an 710071, China (e-mail: wwwang@ @ail.xidian.edu.cn; xcfeng@mail.xidian.edu.cn).

P.-L. Shui is with the National Laboratory of Radar Signal Processing, Xidian University, Xi'an 710071, China (e-mail: plshui@xidian.edu.cn).

Digital Object Identifier 10.1109/LSP.2007.911148
[12] to general weight rules, and a family of weight functions is given. In wavelet domain, images are regarded as functions in the Besov spaces [7], and the $l^{1}$-norm of wavelet coefficients is used as the regularity constraint for noise reduction. Based upon the proposed variational model, the solution of multifocus image fusion and denoising is equivalent to operating the shrinkage rule to the weighted sum of the wavelet coefficients of noisy multifocus images. Weight functions for fusion are determined based upon the local average modulus of intra- and inter-scale wavelet coefficients and the power transforms.

This letter is organized as follows. Section II gives the spatial variational model of joint fusion and denoising of multifocus images and its solution. Section III gives the model in wavelet domain and its solution. Experimental results and performance evaluation are given in Section IV, followed by a brief conclusion.

\section{Spatial VARiational Model}

We are given two noisy multifocus images $u_{1}(\mathbf{x}), u_{2}(\mathbf{x})$ of a scene, where $\mathbf{x}=\left[\mathbf{x}_{1}, \mathbf{x}_{2}\right]^{T} \in \Omega \subset R^{2}$ and $\partial \Omega$ is the boundary of open region $\Omega$. Without loss of generality, $u_{1}(\mathbf{x})$ is near focused and $u_{2}(\mathbf{x})$ is far focused. What we concern here is how to combine the two noisy images into one uniformly focused image $u(\mathbf{x})$ such that all objects in the scene are clear and the noise is significantly reduced.

Based upon the variational model for image denoising [3], [7], [10], we propose the variational model for joint fusion and denoising as follows:

$$
\begin{aligned}
& u=\underset{u \in B V(\Omega)}{\arg \min }\left\{E ( u ) \equiv \int \int _ { \Omega } \left[w_{1}(\mathbf{x})\left(u(\mathbf{x})-u_{1}(\mathbf{x})\right)^{2}\right.\right. \\
& \left.\left.+w_{2}(\mathbf{x})\left(u(\mathbf{x})-u_{2}(\mathbf{x})\right)^{2}\right] d \mathbf{x}+2 \lambda \iint_{\Omega}|\nabla u(\mathbf{x})| d \mathbf{x}\right\}
\end{aligned}
$$

where $w_{1}(\mathbf{x})$ and $w_{2}(\mathbf{x})$ are two nonnegative weight functions satisfying $w_{1}(\mathbf{x})+w_{2}(\mathbf{x})=1, \nabla u(\mathbf{x})$ denotes the gradient operator, $\iint_{\Omega}|\nabla u(\mathbf{x})| d \mathbf{x}$ represents the total variance (TV) of the image $u(\mathbf{x})$ [it is the seminorm of the bounded variant (BV) space], and $\lambda>0$ is a scalar to trade off the fidelity of the solution to the source images and smoothness of the solution. In the model (1), the first term serves for image fusion and is referred to as the fusion term, and the second term serves for image denoising and is referred to as the regularity term. The variational model in (1) is a generalization of the image fusion in pixel domain [1], [11] and the variational model of image denoising [2], [7], [10]. When source images are noise-free, the second term in (1) vanishes. The simplified model gives a new variational interpretation, and its solution is the pixel-wise weighted average of 
two multifocus images. When a single noisy source image $\hat{u}(\mathbf{x})$ is given, the model (1) is reduced to

$$
\begin{array}{r}
u=\underset{u \in B V(\Omega)}{\arg \min }\left\{E(u) \equiv \iint_{\Omega}(u(\mathbf{x})-\hat{u}(\mathbf{x}))^{2} d \mathbf{x}\right. \\
\left.+2 \lambda \iint_{\Omega}|\nabla u(\mathbf{x})| d \mathbf{x}\right\}
\end{array}
$$

which is the classical ROF model [2] in image restoration and the regularity term serves for noise reduction.

As in solving the variational problem in image restoration [2], we use the gradient descendent method to solve the variational problem (1). Its Euler-Lagrange equation is

$$
\begin{array}{r}
w_{1}(\mathbf{x})\left(u(\mathbf{x})-u_{1}(x)\right)+w_{2}(\mathbf{x})\left(u(\mathbf{x})-u_{2}(\mathbf{x})\right)- \\
\lambda \operatorname{div}\left(\frac{\nabla u(\mathbf{x})}{|\nabla u(\mathbf{x})|}\right)=0 .
\end{array}
$$

By introducing a time variable $t$, (3) is embedded into the evolution equation

$$
\begin{array}{r}
\frac{\partial u(\mathbf{x} ; t)}{\partial t}=w_{1}(\mathbf{x})\left(u_{1}(\mathbf{x})-u(\mathbf{x} ; t)\right)+w_{2}(\mathbf{x})\left(u_{2}(\mathbf{x})-u(\mathbf{x} ; t)\right) \\
+\lambda \operatorname{div}\left(\frac{\nabla u(\mathbf{x} ; t)}{|\nabla u(\mathbf{x} ; t)|}\right) .
\end{array}
$$

As a result, the solution of (3) is just the stationary solution of the evolution (4). Its stationary solution is iteratively obtained by the time-forward difference scheme

$$
\begin{aligned}
u^{(n+1)}=u^{(n)}+\Delta t\left[w_{1}\left(u_{1}-u^{(n)}\right)\right. & \left.+w_{2}\left(u_{2}-u^{(n)}\right)\right] \\
& +\lambda d i v\left(\frac{\nabla u^{(n)}}{\left|\nabla u^{(n)}\right|}\right)
\end{aligned}
$$

where the spatial derivatives can be approximated by differences.

In what follows, we deal with selection of the weight functions in (1). The quality of image fusion depends highly on weight functions. For multifocus images, incorrect focusing blurs the detail structures in images, such as edges and textures. Blurring reduces the modulus of the gradients in the incorrect focusing regions. Thus, most the existing references use the gradient-based criteria to determine weight functions. A typical example is the hard decision map using the local average modulus of gradients [9], [12], which can be described as follows:

$$
\begin{aligned}
w_{1}(\mathbf{x}) & = \begin{cases}1, & \text { if }\left|\bar{\nabla} u_{1}(\mathbf{x})\right|>\left|\bar{\nabla} u_{2}(\mathbf{x})\right| \\
0.5, & \text { if }\left|\bar{\nabla} u_{1}(\mathbf{x})\right|=\left|\bar{\nabla} u_{2}(\mathbf{x})\right| \\
0, & \text { elsewise }\end{cases} \\
|\bar{\nabla} u(\mathbf{x})| & \equiv \frac{1}{\# \mathbf{W}} \sum_{\mathbf{z} \in \mathbf{W}}|\nabla u(\mathbf{x}+\mathbf{z})| \\
w_{2}(\mathbf{x}) & =1-w_{1}(\mathbf{x}),
\end{aligned}
$$

where $\nabla u(\mathbf{x})=\left[\left(\partial u(\mathbf{x}) / \partial x_{1}\right),\left(\partial u(\mathbf{x}) / \partial x_{2}\right)\right]$ is the gradient of $u(\mathbf{x})$ at $\mathbf{x},|\bar{\nabla} u(\mathbf{x})|$ is the local average modulus of gradients in window, and $\mathbf{W}$ is a window centered at $\mathbf{0}$. Here, we give a family of weight functions using the local average modulus of gradients and specified by the power $\alpha$

$$
\begin{aligned}
w_{1}^{\alpha}(\mathbf{x}) & \equiv \frac{\left|\bar{\nabla} u_{1}(\mathbf{x})\right|^{\alpha}}{\left|\bar{\nabla} u_{1}(\mathbf{x})\right|^{\alpha}+\left|\bar{\nabla} u_{2}(\mathbf{x})\right|^{\alpha}} \\
w_{2}^{\alpha}(\mathbf{x}) & =1-w_{1}^{\alpha}(\mathbf{x}), \quad \alpha \in[0, \infty) .
\end{aligned}
$$

The weight functions in (7) are a generalization of (6). When $\alpha \rightarrow \infty$, the weight functions approximate the hard decision maps. Unlike (6), the weight functions in (7) generate soft decision maps, which realize both image fusion and noise suppression. It is known that incorrect focusing incurs less degradation of image quality in smooth regions. In these regions, the local average moduli of gradients of two images are competitive. In terms of (7), the values of the weight functions are close to $1 / 2$ in these regions. Since noises in the two images are mutually independent, the average significantly reduces noise in these smooth regions. For edges and textures, the local average modulus of gradients in the correct focused image is often much larger than that in the incorrect focused one. Therefore, the weighted average in terms of (7) incurs less degradation of fusion quality as compared with the hard decision maps in (6).

It is an easy task to extend the model (1) to cases with more than two noisy multifocus images. Moreover, the regularity term based on the TV in the BV space can also be replaced by the norms in other smooth space.

\section{VARIATIONAL MODEL IN WAVELET DOMAIN}

Wavelet provides multiresolution representations of images and has been successfully applied in image fusion [11] and image denoising [4], [7], [8]. Chambolle uses the Besov spaces instead of the BV space and uses the Bosov seminorm instead of the TV in the ROF model (2) [7]. Moreover, it is proved that the solution of the modified model in wavelet domain is equivalent to the simple wavelet shrinkage [8]. Following this idea, we use the Besov space $B_{1,1}^{1}(\Omega)$ instead of the BV space in the model (1). The model (1) becomes

$$
\begin{aligned}
u= & \underset{u \in B_{1,1}^{1}(\Omega)}{\arg \min }\left\{E ( u ) \equiv \int \int _ { \Omega } \left[w_{1}(\mathbf{x})\left(u(\mathbf{x})-u_{1}(\mathbf{x})\right)^{2}\right.\right. \\
& \left.\left.+w_{2}(\mathbf{x})\left(u(\mathbf{x})-u_{2}(\mathbf{x})\right)^{2}\right] d \mathbf{x}+2 \lambda|u(\mathbf{x})|_{B_{1,1}^{1}(\Omega)}\right\} .
\end{aligned}
$$

In what follows, we replace this model in pixel domain by the model in wavelet domain. Let $d^{l, o}(\mathbf{x}), d_{1}^{l, o}(\mathbf{x})$, and $d_{2}^{l, o}(\mathbf{x})$ be the wavelet coefficients of images $u(\mathbf{x}), u_{1}(\mathbf{x})$, and $u_{2}(\mathbf{x})$, respectively, where the superscripts $l$ and $o \in\{L H, H L, H H\}$ label the level and the oriented subbands, and $\mathbf{x}=\{i, j\}$ denotes spatial locations. Here, we use the energy functional based on the wavelet coefficients of the two noisy images instead of the first term of the energy functional in (8). Moreover, the seminorm of $u(\mathbf{x})$ in $B_{1,1}^{1}(\Omega)$ is equivalent to the $l^{1}$-norm of its wavelet coefficients in an orthonormal wavelet base [6], [7]. As a result, the variational model in wavelet domain is formulized as follows:

$$
\begin{aligned}
d^{l, o}(\mathbf{x}) & =\arg \min \left\{\sum _ { l , o } \sum _ { i , j } \left[w_{1}^{l, o}(\mathbf{x})\left(d^{l, o}(\mathbf{x})-d_{1}^{l, o}(\mathbf{x})\right)^{2}\right.\right. \\
& \left.\left.+w_{2}^{l, o}(\mathbf{x})\left(d^{l, o}(\mathbf{x})-d_{2}^{l, o}(\mathbf{x})\right)^{2}\right]+2 \lambda\left|d^{l, o}(\mathbf{x})\right|\right\}
\end{aligned}
$$


where $\lambda$ is a parameter to achieve a trade-off between fusion and denoising. In the model in wavelet domain, the fused and denoised image can be recovered from the estimated wavelet coefficients. Since each term of the summation in (9) is separable and nonnegative, minimizing the summation is equivalent to minimizing each of its terms. It is easy to prove that the problem (9) in a similar manner in [8] has a unique solution

$$
\begin{aligned}
& d^{l, o}(\mathbf{x})=\operatorname{sign}\left\{\hat{d}^{l, o}(\mathbf{x})\right\} \max \left\{\left|\hat{d}^{l, o}(\mathbf{x})\right|-\lambda, 0\right\} \\
& \hat{d}^{l, o}(\mathbf{x})=w_{1}^{l, o}(\mathbf{x}) d_{1}^{l, o}(\mathbf{x})+w_{2}^{l, o}(\mathbf{x}) d_{2}^{l, o}(\mathbf{x}) .
\end{aligned}
$$

The above solution shows that the joint fusion and denoising of multifocus images based on the variational model (8) is the coefficient-based fusion followed by the wavelet shrinkage.

In what follows, we deal with the weight functions $w_{1}^{l, o}(\mathbf{x})$ and $w_{2}^{l, o}(\mathbf{x})$ that are crucial in the fusion stage. As the gradients in pixel domain, the wavelet coefficients reflect local high-frequency information. Incorrect focusing is equivalent to blurring objects out of "focus" using lowpass smoothing filters and then incurs magnitude reduction of the wavelet coefficients to represent these objects. The coefficient-based, window-based, and region-based criteria using the magnitudes or average magnitudes of wavelet coefficients were proposed to determine weight functions for fusion [11]. It is also shown that the region-based criterion exhibit better consistency of fusion image.

Here, we use the region-based criterion. Let the size of the images is $2^{R}$ by $2^{R}$. In the J-level orthonormal decomposition, the coarsest subbands are $2^{R-J}$ by $2^{R-J}$ matrices. In terms of the spatial orientation tree in the wavelet-based image compression [14], we combine the nodes $\left\{(\mathbf{x}, J, o): \mathbf{x}=(i, j)^{T}, o \in\right.$ $\{L H, H L, H H\}\}$ at the coarsest level and their offspring nodes as a subset. In other words, all the wavelet coefficients except lowpass coefficients are partitioned into $4^{R-J}$ subsets, and the indexes of these subsets are defined as follows.

$$
\begin{gathered}
\text { For } \mathbf{x}=[i, j], 0 \leq i, j \leq 2^{R-J}-1 \\
\begin{array}{c}
\mathbf{S}(\mathbf{x}) \equiv\left\{(\mathbf{y}, l, o): \mathbf{y}=(p, q)^{T}, 2^{J-l} i \leq p\right. \\
\\
q \leq 2^{J-l}(i+1)-1,2^{J-l} j \leq q \leq 2^{J-l}(j+1)-1 \\
l=1,2, \cdots J, o \in\{L H, H L, H H\}\} .
\end{array}
\end{gathered}
$$

It is noted that the wavelet coefficients in $\mathbf{S}(\mathbf{x})$ contain high-frequency information of an image in the square $\left[2^{J} i, 2^{J}(i+1)-\right.$ $1] \times\left[2^{J} j, 2^{J}(j+1)-1\right]$. If this region is blurred, then the average magnitude of the wavelet coefficients in $\mathbf{S}(\mathbf{x})$ will decrease. We desire that in each subset $\mathbf{S}(\mathbf{x})$, the weight function has the same value. A family of region-dependent weight functions is defined as follows:

$$
\begin{aligned}
\mathrm{w}_{1, \alpha}^{(l, o)}(\mathbf{S}(\mathbf{x})) & =\frac{\left|\bar{D}_{1}(\mathbf{x})\right|^{\alpha}}{\left|\bar{D}_{1}(\mathbf{x})\right|^{\alpha}+\left|\bar{D}_{2}(\mathbf{x})\right|^{\alpha}}, \quad \alpha \in(0, \infty) \\
\left|\bar{D}_{i}(\mathbf{x})\right| & \equiv \sum_{(\mathbf{y}, l, o) \in \mathbf{S}(\mathbf{x})}\left|d_{i}^{l, o}(\mathbf{y})\right|, \quad i=1,2 .
\end{aligned}
$$

As in (7), the weighted average fusion based on (12) can reduce noise owing to independency of noises in two multifocus images, in particular, in the smooth region of images. The weight functions as $\alpha \rightarrow \infty$ approximate the region-based choose-max (CM) scheme in [11] and the fusion stage lacks the capability of noise reduction. It is notable that the region-based criterion is advantageous for preserving the consistency of fused images [11]. Additionally, the wavelet-based method is of low complexity as (a)

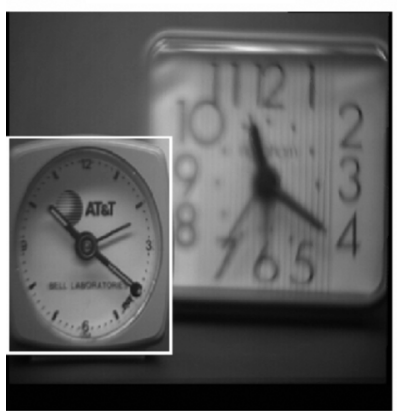

(b)

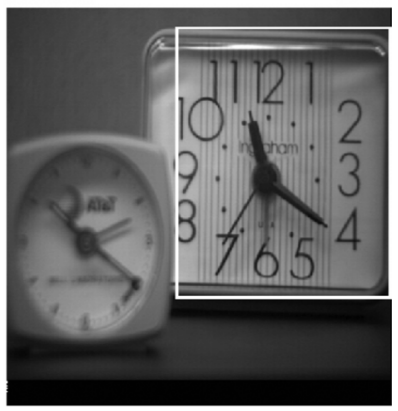

Fig. 1. Near focused image and a far focused image including two clocks. (a) Near focused image. (b) Far focused image.

compared with the variational method in pixel domain, owing to the simple shrinkage rule instead of the iteration algorithm.

\section{EXPERIMENTAL RESULTS}

In the experiments, we use two 512 by 512 8-bit gray-level images. As shown in Fig. 1, one image is near focused where the smaller clock is in "focus" and is clear while the larger clock is out of "focus" and is blurred. The other is far focused, and the situations for the two clocks are just contrary. Therefore, a fused image of high quality should contain both the smaller clock in Fig. 1(a) and the larger clock in Fig. 1(b). In order to quantitatively evaluate the performance of the proposed algorithms, we choose a clear region in each image, as shown in Fig. 1(a) and (b) (where the selected regions are labeled by the white rectangles), to calculate the total mean square error (MSE) of a fused and denoised image in the two regions. In experiments, the two multifocus images are added by computer-generated zero-mean Gaussian white noise, and noise levels are measured using the standard deviations $\sigma$.

In the first set of experiments, we consider the variational method in the pixel domain. It is easily found that the fusion of the two noisy images using the weight functions in (7) results in the fact that noise in the fused image is spatial nonstationary. For each pixel in the fused noisy image, noise standard deviation is determined by the weight functions and the noisy standard deviation in the original noisy images in terms of the following formula:

$$
\sigma_{\alpha}(\mathbf{x})=\sigma \sqrt{1-2 W_{1}^{\alpha}(\mathbf{x}) W_{2}^{\alpha}(\mathbf{x})} .
$$

This is owing to the fact that the noises in the two noisy images are mutually independent. Moreover, the formula (13) shows that the fusion of the two noisy images also reduces noise when $W_{1}^{\alpha}(\mathbf{x}) W_{2}^{\alpha}(\mathbf{x}) \neq 0$. For the fusion based on the choose-max rule in (6), equivalently, $\alpha=\infty$ in (7), the fusion of the two noisy images does not reduce noise because $W_{1}^{\infty}(\mathbf{x}) W_{2}^{\infty}(\mathbf{x})=$ 0 at almost all pixels. The noise average standard deviation in the fused noisy image is

$$
\bar{\sigma}_{\alpha}=\frac{\sigma}{N^{2}} \sum_{\mathbf{x}} \sqrt{1-2 W_{1}^{\alpha}(\mathbf{x}) W_{2}^{\alpha}(\mathbf{x})}
$$

where $N^{2}$ is the number of the pixels in images. As a result, we can use the ratio $\bar{\sigma}_{\alpha} / \sigma$ to measure the capability of noise suppression of the weight functions in (7).

For the variational method in the pixel domain (2), selection of the parameter $\lambda$ is based on the trade-off between the fidelity of the fused image and noise reduction. In the variational 
(a)
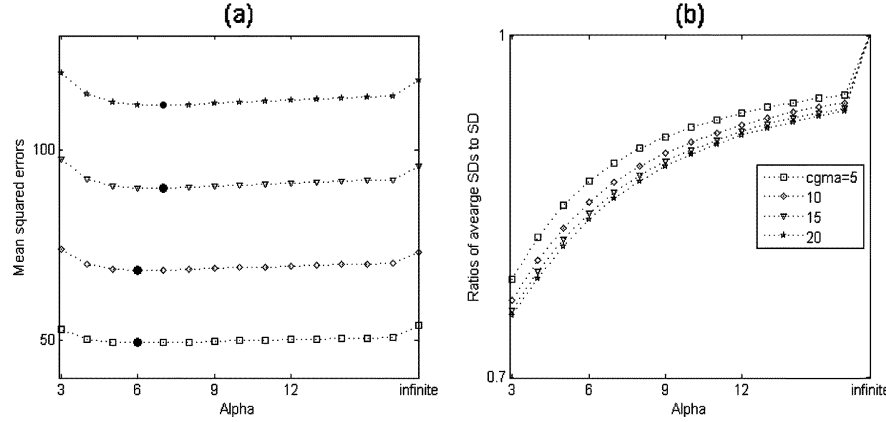

Fig. 2. Performance evaluation of the variational method in pixel domain, where the black circles represent the minimal output mean squared errors for different noise levels in Fig. 2(a). (a) Mean squared errors. (b) Ratios of average SDs to SD.

methods for image denoising [2], [3], the parameter $\lambda$ is selected empirically. Here, we take $\lambda=\bar{\sigma}_{\alpha}$. For the noise levels $\sigma=5$, $10,15,20$, and $\alpha=3,4, \cdots, 16, \infty$, the mean squared errors of fused and denoised regions labeled in Fig. 1(a) and (b) are plotted in Fig. 2(a). In Fig. 2(b), the ratios $\bar{\sigma}_{\alpha} / \sigma$ are shown to demonstrate the capability of noise suppression of weight functions with different $\alpha$ for different noise levels. It can be seen that the capability of noise suppression in the fusion stage degrades as the parameter $\alpha$ increases. The experimental results show that, for the variational method in pixel domain, when $\alpha$ is in the interval [5, 9], the fused and denoised images have satisfactory quality. Particularly, the new weight functions in (7) outperform the choose-max rule in (6) [9], [12] in performance.

In the second set of experiments, we consider the fused and denoised method in the wavelet domain. The five-level decimated decomposition using the linear phase biorthogonal wavelet base "Bior9/7" because simple boundary processing and good spatial localization and smoothness. In fact, other wavelet bases can also be used in this method. In the wavelet shrinkage rules [8], selection of the thresholds $\lambda$ were widely investigated, for example, the universe threshold of Donoho. These thresholds often over-smooth images because removing most of pure noise wavelet coefficients are their primary consideration. Here, we desire that the fused and denoised images can preserve better details. Therefore, we take the threshold to be equal to the average standard deviation of the fused noisy images. As in the pixel domain, the weighted average of the two noisy images in wavelet domain based on the weight functions (12) results in that the noise in the fused image is spatial nonstationary. The average standard deviation can be calculated from the weight functions in terms of

$$
\bar{\sigma}_{\alpha}=\frac{\sigma}{N^{2}} \sum_{(\mathbf{x}, l, o)} \sqrt{1-2 W_{1, \alpha}^{(l, o)}(\mathbf{x}) W_{2, \alpha}^{(l, o)}(\mathbf{x})}
$$

where $N^{2}$ is the number of the wavelet coefficients. For $\sigma=0$, $5,10,15,20$, and $\alpha=1,2, \cdots, 31, \infty$, the mean squared errors of the fused and denoised images are shown in Fig. 2(a), and the ratios of the average noise standard deviations in the fused noisy images to the noise standard deviations of the original images are shown in Fig. 3(b). From Fig. 3(b), it can be seen that the capability of the weight functions in (12) is degraded as $\alpha$ increases. For the choose-max rule in [11], the fusion stage cannot reduce noise in images. It is notable that the incorrect focusing regions in images will have more effect on the fused noisy images when $\alpha$
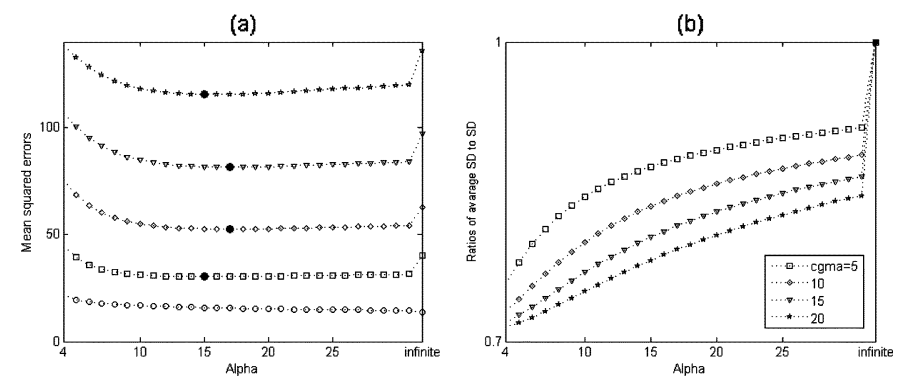

Fig. 3. Performance evaluation of the wavelet-based method, where the black circles represent the minimal output mean squared errors for different noise levels and the white circles denote the noise-free case in Fig. (3a). (a) Mean squared errors. (b) Ratios of average SDs to SD.

becomes smaller. Therefore, selection of $\alpha$ in weight functions is a trade-off between the definition of objects of interest in fused images and the capability of noise suppression in the fusion stage. As shown in Fig. 3(a), when images are noise-free, the choose-max rule in [11] gives the minimal mean squared error; when images are noisy and $\alpha \in[10,25]$, the proposed method achieves better performance than the choose-max rule.

\section{CONCLUSION}

In this letter, the variational models for fusion and denoising of noisy multifocus images are proposed. The algorithms are developed for the case of two noisy multifocus images. It is straightforward to extend the algorithms to the case of noisy multifocus images more than two. The experimental results show also that the algorithm in wavelet domain is slightly better than the algorithm in pixel domain in performance.

\section{REFERENCES}

[1] D. Ishita and C. Bhabatosh, "A simple and efficient algorithm for multifocus image fusion using morphological wavelets,' Signal Proces., vol. 86, pp. 924-936, 2006.

[2] L. Rudin, S. Osher, and E. Fatemi, "Nonlinear total variational based noise removal algorithms," Phys. D, vol. 60, pp. 259-268, 1992.

[3] D. Mumford and J. Shah, "Optimal approximation by piecewise smooth functions and associated variational problems," Comm. Pure. Appl. Math., vol. 42, no. 5, pp. 577-685, 1989.

[4] R. DeVore and B. Lucier, "Fast wavelet techniques for near-optimal image processing," in Proc. IEEE Military Communication Conf. Record San Diego, Piscataway, NJ, 1992, vol. 3, pp. 1129-1135.

[5] R. DeVore and P. Popov, "Interpolation of Besov spaces," Trans. Amer. Math. Soc., vol. 305, pp. 397-414, 1988.

[6] Y. Meyer, Wavelets and Operators. Cambridge, U.K.: Cambridge Univ. Press, 1992.

[7] A. Chambolle, R. DeVore, N. Lee, and B. Lucier, "Nonlinear wavelet image processing: Variational problem, compression and noise removal through wavelet shrinkage," IEEE Trans. Image Process., vol. 7, no. 3, pp. 319-335, Mar. 1998.

[8] D. Donoho and I. Johnstone, "Ideal spatial adaption via wavelet shrinkage," Biometrika, vol. 81, no. 3, pp. 425-455, 1994.

[9] V. Petrovic and C. Xydeas, "Gradient-based multiresolution image fusion,” IEEE Trans. Image Process., vol. 13, no. 2, pp. 228-237, Feb. 2004.

[10] S. Christophe, B. Laure, A. Gilles, and Z. Josiane, "A variational model for image classification and restoration," IEEE Trans. Pattern Anal. Mach. Intell., vol. 22, no. 5, pp. 460-472, May 2000.

[11] P. Gonzalo and J. Cruz, "A wavelet-based image fusion tutorial," Pattern Recognit., vol. 37, no. 9, pp. 1855-1872, 2004.

[12] H. Eltoukhy and S. Kavusi, "A computationally efficient algorithm for multifocus image reconstruction," in Proc. SPIE Electron. Imaging, 2003, vol. 5017, pp. 332-341.

[13] G. Aubert and P. Kornprobst, Mathematical Problems in Image Processing, Applied Mathematical Sciences. New York: Springer, 2002.

[14] A. Said and W. Pearlman, "A new, fast, and efficient image codec based on set partitioning in hierarchical trees," IEEE Trans. Circuits Syst. Video Technol., vol. 6, no. 3, pp. 243-250, Mar. 1996. 